\title{
Efficacy and Safety of Palbociclib and Fulvestrant in Japanese Patients With ER+/HER2- Advanced/Metastatic Breast Cancer
}

\author{
HIROHITO SEKI ${ }^{1}$, TAKASHI SAKURAI ${ }^{1}$, YUKA MAEDA ${ }^{1}$, NAOHIKO OKI ${ }^{1}$, MINA AOYAMA $^{1}$, \\ RYOU YAMAGUCHI ${ }^{1}$, TOSHIKI TOKUDA ${ }^{1}$, TAKUJI KABURAGI ${ }^{1}$, TAKEHIRO OKUMURA ${ }^{1}$, \\ TSUYOSHI KARAHASHI ${ }^{1}$, KENICHIRO NAKAJIMA ${ }^{1}$, KAORI HIGETA ${ }^{2}$ and KEN SHIMIZU ${ }^{3}$ \\ Divisions of ${ }^{1}$ Surgery, ${ }^{2}$ Pharmacy and ${ }^{3}$ Pathology, Saitama Medical Center, Saitama, Japan
}

\begin{abstract}
Background/Aim: Published data have shown that palbociclib-fulvestrant can significantly improve the progressionfree survival (PFS) of estrogen receptor-positive/human epidermal growth factor receptor 2-negative (ER+/HER2-) metastatic breast cancer patients, but not of Japanese patients. We conducted this retrospective study to verify the efficacy and safety of palbociclib-fulvestrant in Japanese patients. Patients and Methods: ER+/HER2- metastatic breast cancer patients treated with fulvestrant $(n=39)$ or palbociclib-fulvestrant $(n=31)$ at the Saitama Medical Center from July 2012 to November 2018 were evaluated. Results: Overall response rates (ORRs) were $2.6 \%$ (fulvestrant) and $41.9 \%$ (palbociclib-fulvestrant) $(p<0.001)$, and clinical benefit rates (CBRs) were $23.1 \%$ and $61.3 \%$ ( $p=0.002$ ), respectively. The palbociclib-fulvestrant group had significantly higher CBR and PFS (hazard ratio(HR):0.272, 95\% confidence interval(95CI):0.128-0.574 for PFS). Grade 3/4 neutropenia occurred in $80.6 \%$ of the palbociclib-fulvestrant group, while febrile neutropenia was not detected. Conclusion: Japanese ER+/HER2- metastatic breast cancer patients tolerated palbociclib-fulvestrant, with significantly improved clinical outcomes.
\end{abstract}

Endocrine therapy for estrogen receptor/human epidermal growth factor receptor 2 (ER+/HER2-) tumours has greatly contributed to reduce early breast cancer recurrence (1). However, some patients relapse during, or following the completion of adjuvant therapy, and metastatic breast cancer (MBC) treatment remains a significant clinical issue.

This article is freely accessible online.

Correspondence to: Hirohito Seki, Division of Surgery, Saitama Medical Center, 4-9-3 Kitaurawa, Urawa-ku, Saitama-city, Saitama, 330-0074, Japan. Tel: +81 488324951 (Ext 1866), Fax: +81 488337527, e-mail: hirohito.seki@gmail.com

Key Words: Metastatic breast cancer, ER positive, palbociclib, CDK4/6 inhibitor, Fulvestrant.
Tamoxifen or aromatase inhibitors are the standard of care for ER+ MBC (2-4). Fulvestrant is a selective ER downregulator, with superior efficacy to aromatase inhibitors for $\mathrm{ER}+\mathrm{MBC}(5,6)$, however, endocrine monotherapy has often offered limited clinical benefit $(7,8)$.

Targeting the molecular components of the cell cycle to interfere with cell-cycle progression is a logical strategy for cancer treatment. Cyclin-dependent kinases (CDK) 4 and 6 promote cell-cycle progression. Palbociclib selectively inhibits CDK4 and CDK6, which ultimately inhibits DNA synthesis $(9,10)$. Palbociclib is an approved novel molecular targeting drug for hormone receptor-positive/HER2advanced or MBC combined with endocrine therapy (11). The phase 2 PALOMA-1 trial has demonstrated the efficacy and safety of palbociclib as first-line therapy combined with letrozole in patients with ER+/HER2- MBC (12). Patients treated with palbociclib-letrozole had significantly longer median progression-free survival (PFS) compared to those treated with letrozole (13). In the phase 3 PALOMA-3 trial, palbociclib-fulvestrant significantly improved PFS in patients with hormone receptor-positive/HER2- MBC who were resistant to endocrine therapy (14-17). Although neutropenia was the most frequent adverse event with palbociclib, the incidence of febrile neutropenia was very low.

A subgroup analysis of Japanese patients in the PALOMA-3 study (18) reported that palbociclib-fulvestrant had no significant effect on PFS compared to placebofulvestrant. In addition, the study showed that there was no significant difference in adverse events between the overall population and Japanese patients, except for a higher rate of neutropenia in Japanese patients receiving palbociclib.

Therefore, we conducted a retrospective study to verify the efficacy and safety of palbociclib-fulvestrant in Japanese patients.

\section{Patients and Methods}

ER+/HER2 - advanced or MBC patients treated with fulvestrant $(n=39)$ or palbociclib-fulvestrant $(n=31)$ at the Saitama Medical Center from July 2012 to November 2018 were included. All patients 
provided informed consent for treatment. This retrospective study was approved by the Institutional Review Board of the Saitama Medical Center (IRB no. 19-2), in accordance with the Declaration of Helsinki and its amendments (19). Fulvestrant, (500 mg, intramuscular) was administered on days 1 and 15 (cycle 1), then every 28 days starting from day 1 of cycle 1 . Palbociclib, (125 mg/day, oral) was administered on days 1-21, followed by 7 days off treatment for every 28-day cycle. Premenopausal and perimenopausal women received subcutaneous injections of an LHRH agonist. The therapeutic effects were evaluated by RECIST guidelines (version 1.1) (20).

Dose interruption/reduction of palbociclib was defined as follows: i) for grade 3 neutropenia or thrombocytopenia, palbociclib was interrupted until recovery to grade $\leq 2$ and then continued at the same dose, ii) for grade 3 neutropenia with fever, palbociclib was interrupted until recovery to grade $\leq 2$ and then resumed with one dose-level reduction, iii) for grade 4 neutropenia or thrombocytopenia, palbociclib was interrupted until recovery to grade $\leq 2$ and then resumed with one dose-level reduction, iv) for grade 3 non-hematologic abnormalities, palbociclib was interrupted until recovery to grade $\leq 2$ and then resumed with one dose-level reduction. Two dose-level reductions in palbociclib were permitted. No dose reductions in fulvestrant were allowed. Laboratory tests were performed every 2 weeks during the first two cycles and on day 1 of subsequent cycles. The severity of adverse events was recorded and graded according to the National Cancer Institute Common Terminology Criteria for Adverse Events (CTCAE v4.0) (21).

Statistical analysis. Statistical analysis was performed using SPSS 23.0 (SPSS Inc., Chicago, IL, USA). Clinicopathlogical variables were compared using Fisher's exact and chi-squared tests. The Mann-Whitney test for categorical variables was used for age analysis. PFS was estimated by Kaplan-Meier with $95 \%$ confidence intervals (CIs). A log-rank test was used to compare PFS between groups. Hazard Ratios (HR) were estimated from the Cox proportional hazards regression models. Values of $p<0.05$ were considered significant.

\section{Results}

Patients' characteristics. Patients from both groups had a median age of 64 years and all had HER2- disease. In the fulvestrant group, $92.3 \%$ were postmenopausal compared to $77.4 \%$ of the palbociclib-fulvestrant group. In the fulvestrant group, i) $79.5 \%$ were ER+ and progesterone receptor $(\mathrm{PgR})+$ compared to $87.1 \%$ in the palbociclib-fulvestrant group, ii) $56.4 \%$ had visceral disease compared to $48.4 \%$ in the palbociclib-fulvestrant group, and iii) $18.0 \%$ had bone disease only compared to $22.6 \%$ in the palbociclibfulvestrant group. The median disease-free intervals (DFIs) were 25.4 months (fulvestrant) and 22.2 months (palbociclibfulvestrant). There was no significant difference in the clinicopathological factors (Table I).

Efficacy. The fulvestrant group had a complete response rate of $0 \%$ compared to $3.2 \%$ for the palbociclib-fulvestrant group; the partial response rate was $2.6 \%$ (fulvestrant) and $38.7 \%$ (palbociclib-fulvestrant). The long stable disease rate was $20.5 \%$ (fulvestrant) compared to $22.6 \%$ (palbociclib-fulvestrant). The overall response rates (ORRs) in the fulvestrant and palbociclibfulvestrant groups were $2.6 \%$ and $41.9 \% \quad(p<0.001)$, respectively. The corresponding clinical benefit rates (CBRs) were $23.1 \%$ and $61.3 \%(p=0.002)$, respectively (Table II).

The correlation between CBR and clinicopathological factors showed significant differences in CBR in patients with the following characteristics: age $<70$ years (fulvestrant: $16.7 \%$ versus palbociclib-fulvestrant:63.2\%, $p=0.002$ ), body mass index $(\mathrm{BMI}) \geq 25$ (fulvestrant:10\% versus palbociclibfulvestrant:69.2\%, $p=0.001), \leq 1$ previous line of endocrine therapy (fulvestrant: $21.1 \%$ versus palbociclib-fulvestrant: $60 \%$, $p=0.001$ ),$\leq 1$ previous line of chemotherapy (fulvestrant:21.1\% versus palbociclib-fulvestrant: $61.5 \% ; p=0.002)$, no sensitivity to prior endocrine therapy (fulvestrant: $22.5 \%$ versus palbociclib-fulvestrant:60\%, $p=0.0049$ ), stage I-III at initial diagnosis (fulvestrant: $23.3 \%$ versus palbociclib-fulvestrant: $59.1 \%, p=0.011$ ), DFI $\geq 24$ months (fulvestrant:30\% versus palbociclib-fulvestrant:69.2\%, $p=0.038$ ), two or more metastatic sites (fulvestrant: $21.7 \%$ versus palbociclibfulvestrant: $65 \%, p=0.006$ ), and visceral metastasis (fulvestrant:22.7\% versus palbociclib-fulvestrant:73.3\%, $p=0.006$ ) (Table III). The palbociclib-fulvestrant group had significantly improved median PFS (13.3 months) compared to the fulvestrant group (3.9 months) [Hazard Ratio (HR):0.272, 95\% CI:0.128-0.574, log-rank test $p<0.001]$ (Figure 1).

Adverse events. The most common adverse events in the palbociclib/fulvestrant group were leukopenia, neutropenia, anaemia, and fatigue (Table IV). More frequent hematological adverse events occurred in the palbociclib/ fulvestrant group. No group experienced febrile neutropenia. The most common non-hematological adverse events were fatigue $(41.9 \%$ in palbociclib-fulvestrant versus $5.2 \%$ in fulvestrant). Two patients experienced fever without neutropenia in the palbociclib-fulvestrant group. The only $>$ grade 3 non-hematological adverse event was liver dysfunction $(5.1 \%)$, which occurred in the fulvestrant group. There were no serious adverse events in either group.

There was no dose discontinuation of palbociclib due to adverse events; however, 58.1\% (18/31) required dose interruption and $71 \%(18 / 31)$ required dose reduction due to grade $3 / 4$ neutropenia. Sixteen $(51.6 \%)$ patients required one dose-level reduction and $6(19.4 \%)$ required two dose-level reductions. The median number of courses for the first dose reduction was 2 (range:1-5), and the median for the second dose reduction was 3 (range:2-5).

\section{Discussion}

ER+/HER2- MBC treatment has remarkably changed over the past few decades. Aromatase inhibitors have shown effectiveness compared to tamoxifen in postmenopausal women with MBC $(3,4)$. Subsequently, the selective ER 
Table I. Patients' characteristics.

\begin{tabular}{|c|c|c|c|}
\hline & $\begin{array}{c}\text { Fulvestrant } \\
\text { alone } \\
(\mathrm{n}=39)\end{array}$ & $\begin{array}{l}\text { Palbociclib- } \\
\text { fulvestrant } \\
\quad(\mathrm{n}=31)\end{array}$ & $p$-Value \\
\hline \multicolumn{4}{|l|}{ Age (years) } \\
\hline Median (range) & $64(44-89)$ & $64(30-87)$ & 0.522 \\
\hline$<70$ years & 30 & 19 & 0.194 \\
\hline$\geq 70$ years & 9 & 12 & \\
\hline Menopausal status & & & 0.096 \\
\hline Premenopausal & 3 & 7 & \\
\hline Postmenopausal & 36 & 24 & \\
\hline BMI & & & 0.436 \\
\hline$<25$ & 23 & 18 & \\
\hline$\geq 25$ & 10 & 13 & \\
\hline ECOG performance status & & & 1.000 \\
\hline 0 & 37 & 31 & \\
\hline 1 & 1 & 0 & \\
\hline Pathological subtype & & & 0.118 \\
\hline IDC & 31 & 30 & \\
\hline ILC & 4 & 0 & \\
\hline Unknown & 5 & 1 & \\
\hline Hormone-Receptor status & & & 0.745 \\
\hline ER-positive and PR positive & 31 & 27 & \\
\hline ER-positive and PR negative & 6 & 4 & \\
\hline Unknown & 2 & 0 & \\
\hline Ki-67 & & & 0.062 \\
\hline$<40 \%$ & 15 & 6 & \\
\hline$\geq 40 \%$ & 8 & 12 & \\
\hline Unknown & 16 & 13 & \\
\hline Nuclear grade & & & 0.23 \\
\hline 1 or 2 & 22 & 18 & \\
\hline 3 & 1 & 1 & \\
\hline Unknown & 16 & 12 & \\
\hline Disease stage at initial diagnosis & & & 0.708 \\
\hline I & 3 & 3 & \\
\hline II & 14 & 11 & \\
\hline III & 11 & 5 & \\
\hline IV & 9 & 9 & \\
\hline Unknown & 2 & 3 & \\
\hline \multicolumn{4}{|l|}{ Metastatic site } \\
\hline Visceral & 22 & 15 & 0.631 \\
\hline Nonvisceral & 20 & 19 & 0.472 \\
\hline Bone only & 7 & 7 & 0.766 \\
\hline
\end{tabular}

down-regulator, fulvestrant, has been found to be significantly better PFS compared to aromatase inhibitors for postmenopausal women with ER+/HER2- MBC (6). Endocrine therapy has been a standard treatment strategy for ER+/HER2- MBC patients without a critical condition (22). Interestingly, endocrine therapy combined with a CDK4/6 inhibitor can significantly improve PFS compared to endocrine monotherapy, thus it has become a standard of treatment for ER+/HER2- MBC (13-15, 17, 23-25).

In a phase 3 trial, palbociclib-fulvestrant did not improve PFS in Japanese patients with ER+/HER2- MBC. The

\begin{tabular}{|c|c|c|c|}
\hline & $\begin{array}{c}\text { Fulvestrant } \\
\text { alone } \\
(\mathrm{n}=39)\end{array}$ & $\begin{array}{l}\text { Palbociclib- } \\
\text { fulvestrant } \\
\quad(\mathrm{n}=31)\end{array}$ & $p$-Value \\
\hline No. of metastatic sites & & & 0.154 \\
\hline 1 & 16 & 11.0 & \\
\hline 2 & 9 & 11 & \\
\hline 3 & 11 & 2 & \\
\hline$\geq 4$ & 3 & 7 & \\
\hline \multicolumn{4}{|l|}{ Disease-free interval (month) } \\
\hline Median (range) & $\begin{array}{c}25.4 \\
(0.5-138)\end{array}$ & $\begin{array}{c}22.2 \\
(0.5-22.2)\end{array}$ & 0.558 \\
\hline$\leq 24 \mathrm{mo}$ & 13 & 8 & 1 \\
\hline$>24 \mathrm{mo}$ & 19 & 13 & \\
\hline Prior endocrine therapy & & & - \\
\hline AI with or without GnRH agonist & 35 & 23 & \\
\hline TAM with or without GnRH agonist & 12 & 7 & \\
\hline Toremifen & 9 & 9.0 & \\
\hline Fluvestrant & 0 & 4.0 & \\
\hline mTOR inhibitor & 2 & 4 & \\
\hline Prior chemotherapy & & & - \\
\hline Taxane & 4 & 5 & \\
\hline Anthracycline & 1 & 2 & \\
\hline Eriburin & 3 & 5 & \\
\hline Vinorelbine & 0 & 1 & \\
\hline Oral 5FU & 2 & 1 & \\
\hline Most recent therapy & & & 0.193 \\
\hline None & 1 & 3 & \\
\hline Endocrine & 30 & 21 & \\
\hline Chemo & 6 & 3 & \\
\hline mTOR inhibitor & 2 & 1 & \\
\hline CDK4/6 inhibitor & 0 & 3 & \\
\hline $\begin{array}{l}\text { Previous lines of therapy in } \\
\text { the context of metastatic disease }\end{array}$ & & & 0.539 \\
\hline 0 & 1 & 3 & \\
\hline 1 & 12 & 11 & \\
\hline 2 & 9 & 5 & \\
\hline$\geq 3$ & 17 & 12 & \\
\hline
\end{tabular}

BMI: Body mass index; ECOG: Eastern Cooperative Oncology Group; IDC: invasive ductal carcinoma; ILC: invasive lobular carcinoma; ER: estrogen receptor; PgR: progesterone receptor; $\mathrm{AI}$ : aromatase inhibitor; GnRH: gonadotropin releasing hormone; TAM: tamoxifen; mTOR: mammallian target of rapamycin; 5FU: 5-fluorouracil; CDK: cyclindependent kinase.

frequency of grade 3/4 neutropenia was higher in Japanese patients than the overall population (18). Therefore, it was necessary to verify the efficacy and safety of palbociclibfulvestrant for Japanese patients with ER+/HER2- MBC.

The palbociclib-fulvestrant group had significantly better ORR and CBR compared to the fulvestrant group. In the palbociclib-fulvestrant group of the PALOMA-3 trial, the ORRs were $21 \%$ in the overall population and $18.5 \%$ in the Japanese subgroup, and the CBRs were $66.3 \%$ in the overall population and $74.1 \%$ in the Japanese subgroup (18). The CBR in this study was like the trial, but the current study had 
Table II. Comparison of overall response rate in the fulvestrant alone and palbociclib-fulvestrant groups.

Fulvestrant Palbociclib- $p$-Value alone fulvestrant

$(n=39) \quad(n=31)$

\begin{tabular}{lccr}
\hline Response & & & \\
CR & 0 & 1 & \\
PR & 1 & 12 & \\
LSD & 8 & 7 & \\
SD & 4 & 2 & \\
PD & 26 & 9 & \\
RR & $2.6 \%$ & $41.9 \%$ & $<0.001$ \\
CBR & $23.1 \%$ & $61.3 \%$ & 0.002 \\
\hline
\end{tabular}

CR: Complete response; PR: partial response; LSD: long stable disease; SD: stable disease; PD: partial response; RR: response rate; $\mathrm{CBR}$ : clinical benefit rate.

a better ORR. However, there were fewer patients with visceral metastasis than in the PALOMA-3 trial (48\% and $63 \%$, respectively). Our result indicated better clinical response with palbociclib-fulvestrant than fulvestrant for Japanese patients with ER+HER2- MBC (18).

We observed higher CBRs: i) in patients aged $<70$ years, ii) with $\mathrm{BMI} \geq 25$, iii) $\mathrm{PgR}$ positivity, iv) stage I-III at initial diagnosis, v) DFI 24 months or longer, vi) $\leq 1$ previous line of endocrine therapy, vii) $\leq 1$ previous line of chemotherapy, viii) no sensitivity to prior endocrine therapy, ix) two or more metastatic sites, and $\mathrm{x}$ ) visceral metastasis in the palbociclib-fulvestrant group.

It has been previously shown that there was significantly improved median PFS with palbociclib-fulvestrant versus fulvestrant in the <65-year old subgroup and 65-74-year old subgroup, but no significant improvement in the $\geq 75$-year old subgroup (23). Our study also reports that there is no significant difference in CBR between fulvestrant and palbociclibfulvestrant in the $\geq 70$-year old subgroup, suggesting that fulvestrant provides a sufficient benefit for elderly patients.

CBR in the palbociclib-fulvestrant group was significantly better compared to the fulvestrant group in patients with a BMI of $\geq 25$. A previous study has demonstrated that obesity is a risk factor for postmenopausal ER+ breast cancer (26). In fact, the efficacy of endocrine therapy for ER+ postmenopausal MBC is significantly worse for patients with a BMI of $\geq 25$ than for those with a BMI $<25 \mathrm{~kg} / \mathrm{m}^{2}(27)$. Although no direct evidence exists for the relationship between the efficacy of CDK4/6 inhibitor and obesity, endocrine therapy alone is less effective for obese patients, which may explain the improved CBR when combined endocrine therapy and palbociclib is administered.

Patients with stage I-III disease at initial diagnosis and DFI >24 months had significantly better CBR with

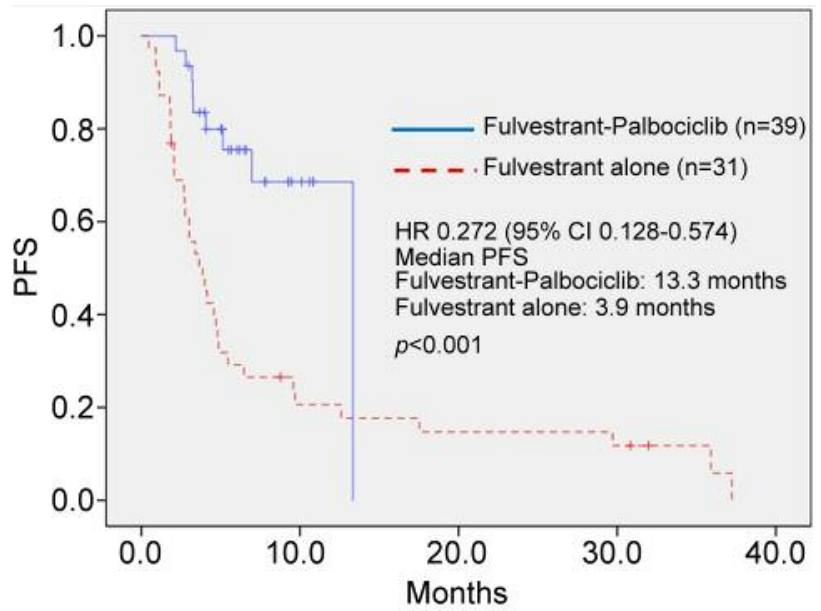

Figure 1. Kaplan-Meier curve for PFS in patients with ER+/HER2$M B C$. Cox's proportional hazard ratio (95\%CI):0.272 (range:0.1280.574). PFS: Progression-free survival; ER: estrogen receptor; HER2: human epidermal growth factor receptor 2; MBC: metastatic breast cancer; CI: confidence interval.

palbociclib-fulvestrant compared to fulvestrant. This finding is supported in the PALOMA-2 trial, where patients receiving palbociclib-fulvestrant had longer PFS in the recurrent subgroup compared to the newly metastatic subgroup (13), and in the PALOMA-3 trial, where the palbociclib-fulvestrant group had significantly more OS in the DFI $>24$ months subgroup (24).

Increased PFS has been reported in patients receiving palbociclib-fulvestrant comparedto fulvestrant, regardless of the number of previous endocrine therapy treatments. For example, patients who were administered palbociclibfulvestrant having received 1 previous line of endocrine therapy had longer PFS compared to patients who received $\geq 2$ previous lines (16). In this study, PFS was significantly longer in patients receiving $\leq 1$ previous line of endocrine therapy compared to those who received $\geq 2$ lines. This suggests a possible benefit for ER+/HER2-MBC patients to receive palbociclib-fulvestrant as an early treatment, prior to the application of multiple lines of endocrine therapy.

Palbociclib-fulvestrant has previously led to a significantly better prognosis compared to fulvestrant in patients with and without sensitivity to prior endocrine therapy (14). In this study, palbociclib-fulvestrant also resulted in a significantly better CBR in patients without sensitivity to prior endocrine therapy. Possibly, palbociclib-fulvestrant contributes to endocrine therapy resistance in MBC patients.

In the PALOMA-3 trial, the PFS in the palbociclibfulvestrant group was significantly longer compared to the fulvestrant group in patients with two or more metastatic sites (16). PFS was also significantly better, regardless of 
Table III. Correlation between CBR and clinicopathological factors in fulvestrant alone and palbociclib-fulvestrant treated patients.

\begin{tabular}{|c|c|c|c|c|}
\hline & Fulvestrant alone (\%) & Palbocicilb-fulvestrant (\%) & OR $(95 \% \mathrm{CI})$ & $p$-Value \\
\hline \multicolumn{5}{|l|}{ Age } \\
\hline$<70$ & 16.7 & 63.2 & $2.656(2.248-32.667)$ & 0.002 \\
\hline$\geq 70$ & 44.4 & 58.3 & $1.375(0.507-3.729)$ & 0.67 \\
\hline \multicolumn{5}{|c|}{ Menopausal status } \\
\hline Pre/Peri & 0 & 85.7 & - & 0.033 \\
\hline Post & 25 & 54.2 & $1.737(1.010-2.985)$ & 0.03 \\
\hline \multicolumn{5}{|l|}{ BMI } \\
\hline$<25$ & 26.1 & 55.6 & $1.813(0.950-13.198$ & 0.105 \\
\hline$\geq 25$ & 10 & 69.2 & $6.923(1.041-46.027)$ & 0.01 \\
\hline \multicolumn{5}{|l|}{ PgR } \\
\hline Negative & 16.7 & 0 & - & 1 \\
\hline Positive & 22.6 & 70.4 & $2.786(1.434-5.412$ & $<0.001$ \\
\hline \multicolumn{5}{|c|}{ Disease stage at initial diagnosis } \\
\hline I-III & 23.3 & 59.1 & $2.054(1.088-3.877)$ & 0.011 \\
\hline IV & 22.2 & 66.7 & $2.800(0.789-9.940)$ & 0.153 \\
\hline \multicolumn{5}{|c|}{ Disease-free interval (month) } \\
\hline$\leq 24$ & 8.3 & 50 & $3.750(0.635-22.142)$ & 0.109 \\
\hline$>24$ & 30 & 69.2 & $1.912(0.975-3.749)$ & 0.038 \\
\hline \multicolumn{5}{|c|}{$\begin{array}{l}\text { Previous lines of endocrine therapy } \\
\text { in the context of metastatic disease }\end{array}$} \\
\hline 0 or 1 & 21.1 & 60 & $1.771(1.048-2.993)$ & 0.01 \\
\hline$\geq 2$ & 100 & 62.5 & - & 1 \\
\hline \multicolumn{5}{|c|}{$\begin{array}{l}\text { Previous lines of chemo therapy } \\
\text { in the context of metastatic disease }\end{array}$} \\
\hline 0 or 1 & 21.1 & 61.5 & $2.250(1.243-4.073)$ & 0.002 \\
\hline$\geq 2$ & 100 & 60 & - & 1 \\
\hline \multicolumn{5}{|c|}{ Prior Sensitivity to endocrine therapy } \\
\hline No & 22.6 & 60 & $1.592(0.941-2.692)$ & 0.049 \\
\hline Yes & 33.3 & 58.3 & $2.000(0.482-8.306)$ & 0.62 \\
\hline \multicolumn{5}{|c|}{ No. of Metastatic sites } \\
\hline 1 & 25 & 54.5 & $1.765(0.778-4.002)$ & 0.224 \\
\hline$\geq 2$ & 21.7 & 65 & $2.592(1.183-5.677)$ & 0.006 \\
\hline \multicolumn{5}{|l|}{ Metastatic site } \\
\hline Viceral & 22.7 & 73.3 & $2.590(1.217-5.516)$ & 0.006 \\
\hline Non-viceral & 23.5 & 50 & $1.857(0.780-4.422)$ & 0.157 \\
\hline Bone only & 28.6 & 71.4 & $2.500(0.708-8.827)$ & 0.286 \\
\hline
\end{tabular}

CBR: Clinical benefit rate; OR: odds ratio; CI,: confidence interval; BMI: body mass index; PgR: progesterone receptor.

visceral metastasis (24). In this study, better CBR in the palbociclib-fulvestrant group was observed in patients with multiple metastatic sites and those with visceral metastasis, suggesting that palbociclib-fulvestrant therapy has more clinical benefit in patients with extensive metastatic disease.

Our results suggest that the most appropriate cases for treatment with palbociclib-fulvestrant are: i) aged $<70$, ii) obese, iii) PgR positive, iv) with DFI >24 months, v) earlyline treatment with resistance to prior endocrine therapy, and vi) multiple metastases, including visceral metastasis. Endocrine monotherapy can have sufficient clinical benefit for elderly patients with mild metastatic disease.

In a previous report, although the median PFS of the palbociclib-fulvestrant group was significantly longer
Table IV. Dose discontinuation, interruption, and reduction of palbociclib treatment.

\begin{tabular}{lcc}
\hline & $\mathrm{N}$ & $\%$ \\
\hline Dose discontinuation & 0 & 0 \\
Dose interruption & 18 & 58.1 \\
Dose reduction (Total) & 22 & 71 \\
1 dose-level reduction & 16 & 51.6 \\
2 dose-level reduction & 6 & 19.4 \\
Course for dose reduction & & \\
1 dose-level reduction & $2(1-5)$ & \\
Median (minimun-max) & & \\
2 dose-level reduction & $3(2-5)$ & \\
Median (minimun-max) & & \\
\hline
\end{tabular}


compared to the fulvestrant group in the entire population, Japanese patients in both groups had no significant difference in PFS (18). In our study, the median PFS was significantly improved in the palbociclib-fulvestrant group. Our result suggests a possibly significant improvement in the prognosis of Japanese patients receiving palbociclibfulvestrant.

Japanese and other Asian patients have lower baseline neutrophil counts compared to non-Asian patients (18). This may explain the higher rate of neutropenia in Japanese patients treated with palbociclib. This higher incidence was not related to a higher palbociclib exposure, lower body weight, lower body surface area/BMI, or older age. The rate of grade 4 neutropenia in the palbociclib-fulvestrant group was $16.1 \%$. This is similar to the PALOMA-3 trial, that reported $26 \%$ grade 4 neutropenia in Japanese patients receiving palbociclib, which was higher compared to the overall population (9\%) (18). Neutropenia caused by palbociclib should not be viewed or managed the same way as neutropenia caused by chemotherapy. A previous study has reported that bone marrow suppression due to palbociclib is not associated with apoptosis, DNA damage response, or cell senescence in vitro (28). Patients treated with palbociclib recover human bone marrow mononuclear cell counts on day 9 following administration; however, this is not observed in patients treated with cytotoxic chemotherapeutic agents. Possibly, bone marrow suppression from palbociclib may less likely cause serious side effects, because patients recover sooner following treatment interruption compared to those treated by chemotherapy.

Although the rate of grade $3 / 4$ neutropenia in this study was $80.6 \%$, there was no febrile neutropenia or treatment discontinuation. A previous study has demonstrated that patients recover more quickly from neutropenia caused by palbociclib compared to neutropenia caused by chemotherapy (28). Approximately $50 \%$ of the palbociclibfulvestrant group in this study had one dose-level reduction, and approximately $20 \%$ had two dose-level reductions. This supports the results of the PALOMA-3 trial, where $44 \%$ of the palbociclib-fulvestrant group and $15 \%$ of the Japanese subgroup had one dose-level reduction (18). The median number of courses of one dose-level reduction in palbociclibfulvestrant in this study was two. In another study, PFS was similar between patients requiring a dose reduction and those who did not, indicating that palbociclib dose reduction does not affect prognosis (29). These results suggest that neutropenia due to palbociclib is an unlikely cause of febrile neutropenia if dose reduction is performed early, and the feasibility of palbociclib in Japanese breast cancer patients is equal to the entire population.

This study had certain limitations. This is a singleinstitution retrospective study with a limited sample size and a short observation period. However, this study indicated a possibility that palbociclib-fulvestrant is effective and well tolerated in Japanese patients with ER+/HER2- MBC. Further studies are required to evaluate long-term prognosis with more cases of this particular patient population.

\section{Conflicts of Interest}

The Authors report no conflicts of interest related to this study.

\section{Authors' Contribution}

Drafting of the manuscript was done by HS and TS.. Literature search and analysis were done by HS, TT, TK, T.O, TK, KN;. Data extraction was done by HS, YM, NO, MA, RY, KH, KS. Manuscript editing was done by HS.

\section{Acknowledgements}

This research did not receive any specific grant from funding agencies in the public, commercial, or not-for-profit sectors.

\section{References}

1 (EBCTCG) EBCTCG: Effects of chemotherapy and hormonal therapy for early breast cancer on recurrence and 15-year survival: An overview of the randomised trials. Lancet 365(9472): 1687-1717, 2005. PMID: 15894097. DOI: 10.1016/ S0140-6736(05)66544-0

2 Klijn JG, Blamey RW, Boccardo F, Tominaga T, Duchateau L and Sylvester R: Combined tamoxifen and luteinizing hormonereleasing hormone (lhrh) agonist versus lhrh agonist alone in premenopausal advanced breast cancer: A meta-analysis of four randomized trials. J Clin Oncol 19(2): 343-353, 2001. PMID: 11208825. DOI: $10.1200 / J C O .2001 .19 .2 .343$

$3 \mathrm{Xu} \mathrm{HB}$, Liu YJ and Li L: Aromatase inhibitor versus tamoxifen in postmenopausal woman with advanced breast cancer: A literature-based meta-analysis. Clin Breast Cancer 11(4): 246251, 2011. PMID: 21737354.DOI: 10.1016/j.clbc.2011.06.003

4 Mauri D, Pavlidis N, Polyzos NP and Ioannidis JP: Survival with aromatase inhibitors and inactivators versus standard hormonal therapy in advanced breast cancer: Meta-analysis. J Natl Cancer Inst 98(18): 1285-1291, 2006. PMID: 16985247. DOI: 10.1093/ jnci/djj357

5 Ellis MJ, Llombart-Cussac A, Feltl D, Dewar JA, Jasiowka M, Hewson N, Rukazenkov Y and Robertson JF: Fulvestrant 500 $\mathrm{mg}$ versus anastrozole $1 \mathrm{mg}$ for the first-line treatment of advanced breast cancer: Overall survival analysis from the phase ii first study. J Clin Oncol 33(32): 3781-3787, 2015. PMID: 26371134. DOI: $10.1200 /$ JCO.2015.61.5831

6 Robertson JFR, Bondarenko IM, Trishkina E, Dvorkin M, Panasci L, Manikhas A, Shparyk Y, Cardona-Huerta S, Cheung KL, Philco-Salas MJ, Ruiz-Borrego M, Shao Z, Noguchi S, Rowbottom J, Stuart M, Grinsted LM, Fazal M and Ellis MJ: Fulvestrant $500 \mathrm{mg}$ versus anastrozole $1 \mathrm{mg}$ for hormone receptor-positive advanced breast cancer (falcon): An international, randomised, double-blind, phase 3 trial. Lancet 388(10063): 2997-3005, 2016. PMID: 27908454. DOI: 10.1016/ S0140-6736(16)32389-3 
7 Baselga J, Campone M, Piccart M, Burris HA, 3rd, Rugo HS Sahmoud T, Noguchi S, Gnant M, Pritchard KI, Lebrun F, Beck JT, Ito Y, Yardley D, Deleu I, Perez A, Bachelot T, Vittori L, Xu Z, Mukhopadhyay P, Lebwohl D and Hortobagyi GN: Everolimus in postmenopausal hormone-receptor-positive advanced breast cancer. N Engl J Med 366(6): 520-529, 2012. PMID: 22149876. DOI: 10.1056/NEJMoa1109653

8 Klijn JG, Beex LV, Mauriac L, van Zijl JA, Veyret C, Wildiers J, Jassem J, Piccart M, Burghouts J, Becquart D, Seynaeve C, Mignolet $\mathrm{F}$ and Duchateau L: Combined treatment with buserelin and tamoxifen in premenopausal metastatic breast cancer: A randomized study. J Natl Cancer Inst 92(11): 903-911, 2000. PMID: 10841825. DOI: 10.1093/jnci/92.11.903

9 Fry DW, Harvey PJ, Keller PR, Elliott WL, Meade M, Trachet E, Albassam M, Zheng X, Leopold WR, Pryer NK and Toogood PL: Specific inhibition of cyclin-dependent kinase $4 / 6$ by pd 0332991 and associated antitumor activity in human tumor xenografts. Mol Cancer Ther 3(11): 1427-1438, 2004. PMID: 15542782 .

10 Marzec M, Kasprzycka M, Lai R, Gladden AB, Wlodarski P, Tomczak E, Nowell P, Deprimo SE, Sadis S, Eck S, Schuster SJ, Diehl JA and Wasik MA: Mantle cell lymphoma cells express predominantly cyclin d1a isoform and are highly sensitive to selective inhibition of cdk4 kinase activity. Blood 108(5): 17441750, 2006. PMID: 16690963. DOI: 10.1182/blood-2006-04016634

11 DeMichele A, Clark AS, Tan KS, Heitjan DF, Gramlich K, Gallagher M, Lal P, Feldman M, Zhang P, Colameco C, Lewis D, Langer M, Goodman N, Domchek S, Gogineni K, Rosen M, Fox K and O'Dwyer P: Cdk 4/6 inhibitor palbociclib (pd0332991) in rb+ advanced breast cancer: Phase ii activity, safety, and predictive biomarker assessment. Clin Cancer Res 21(5): 995-1001, 2015. PMID: 25501126. DOI: 10.1158/10780432.CCR-14-2258

12 Finn RS, Crown JP, Lang I, Boer K, Bondarenko IM, Kulyk SO, Ettl J, Patel R, Pinter T, Schmidt M, Shparyk Y, Thummala AR, Voytko NL, Fowst C, Huang X, Kim ST, Randolph S and Slamon DJ: The cyclin-dependent kinase 4/6 inhibitor palbociclib in combination with letrozole versus letrozole alone as first-line treatment of oestrogen receptorpositive, her2-negative, advanced breast cancer (paloma1/trio-18): A randomised phase 2 study. Lancet Oncol 16(1): 25-35, 2015. PMID: 25524798. DOI: 10.1016/S14702045(14)71159-3

13 Finn RS, Martin M, Rugo HS, Jones S, Im SA, Gelmon K, Harbeck N, Lipatov ON, Walshe JM, Moulder S, Gauthier E, Lu DR, Randolph S, Dieras V and Slamon DJ: Palbociclib and letrozole in advanced breast cancer. N Engl J Med 375(20): 1925-1936, 2016. PMID: 27959613. DOI: 10.1056/NEJMoa 1607303

14 Turner NC, Ro J, Andre F, Loi S, Verma S, Iwata H, Harbeck N, Loibl S, Huang Bartlett C, Zhang K, Giorgetti C, Randolph S, Koehler $\mathrm{M}$ and Cristofanilli M: Palbociclib in hormone-receptorpositive advanced breast cancer. N Engl J Med 373(3): 209-219, 2015. PMID: 26030518. DOI: 10.1056/NEJMoa1505270

15 Loibl S, Turner NC, Ro J, Cristofanilli M, Iwata H, Im SA, Masuda N, Loi S, Andre F, Harbeck N, Verma S, Folkerd E, Puyana Theall K, Hoffman J, Zhang K, Bartlett CH and Dowsett M: Palbociclib combined with fulvestrant in premenopausal women with advanced breast cancer and prior progression on endocrine therapy: Paloma-3 results. Oncologist 22(9): 10281038, 2017. PMID: 28652278. DOI: 10.1634/theoncologist. 2017-0072

16 Cristofanilli M, Turner NC, Bondarenko I, Ro J, Im SA, Masuda N, Colleoni M, DeMichele A, Loi S, Verma S, Iwata H, Harbeck $\mathrm{N}$, Zhang K, Theall KP, Jiang Y, Bartlett CH, Koehler M and Slamon D: Fulvestrant plus palbociclib versus fulvestrant plus placebo for treatment of hormone-receptor-positive, her2negative metastatic breast cancer that progressed on previous endocrine therapy (paloma-3): Final analysis of the multicentre, double-blind, phase 3 randomised controlled trial. Lancet Oncol 17(4): 425-439, 2016. PMID: 26947331. DOI: 10.1016/S14702045(15)00613-0

17 Turner NC, Slamon DJ, Ro J, Bondarenko I, Im SA, Masuda N, Colleoni M, DeMichele A, Loi S, Verma S, Iwata H, Harbeck N, Loibl S, Andre F, Puyana Theall K, Huang X, Giorgetti C, Huang Bartlett $\mathrm{C}$ and Cristofanilli M: Overall survival with palbociclib and fulvestrant in advanced breast cancer. N Engl J Med 379(20): 1926-1936, 2018. PMID: 30345905. DOI: 10.1056/NEJMoa1810527

18 Masuda N, Inoue K, Nakamura R, Rai Y, Mukai H, Ohno S, Hara F, Mori Y, Hashigaki S, Muramatsu Y, Nagasawa T, Umeyama $\mathrm{Y}$, Huang $\mathrm{X}$ and Iwata $\mathrm{H}$ : Palbociclib in combination with fulvestrant in patients with hormone receptor-positive, human epidermal growth factor receptor 2-negative advanced breast cancer: Paloma-3 subgroup analysis of japanese patients. Int J Clin Oncol 24(3): 262-273, 2019. PMID: 30392115. DOI: 10.1007/s10147-018-1359-3

19 World Medical A: World medical association declaration of helsinki. Ethical principles for medical research involving human subjects. Bull World Health Organ 79(4): 373-374, 2001. PMID:11357217.

20 Eisenhauer EA, Therasse P, Bogaerts J, Schwartz LH, Sargent D, Ford R, Dancey J, Arbuck S, Gwyther S, Mooney M, Rubinstein L, Shankar L, Dodd L, Kaplan R, Lacombe D and Verweij J: New response evaluation criteria in solid tumours: Revised recist guideline (version 1.1). Eur J Cancer 45(2): 228247, 2009. DOI: 10.1016/j.ejca.2008.10.026.

21 US Department of Health and Human Services UFaDA CfDEaR, Center for Biologics Evaluation and Research. Guidance for industry: Clinical trial endpoints for the approval of cancer drugs and biogics. FDA, Maryland http://www.fda.gov/downloads/ Drugs/Guidance/Guidances/ucm071590.pdf.

22 Hortobagyi GN: Treatment of breast cancer. N Engl J Med 339(14): 974-984, 1998. PMID: 9753714. DOI: 10.1056/ NEJM199810013391407

23 Rugo HS, Turner NC, Finn RS, Joy AA, Verma S, Harbeck N, Masuda N, Im SA, Huang X, Kim S, Sun W, Iyer S, Schnell P, Bartlett $\mathrm{CH}$ and Johnston S: Palbociclib plus endocrine therapy in older women with hr+/her2- advanced breast cancer: A pooled analysis of randomised paloma clinical studies. Eur J Cancer 101: 123-133, 2018. PMID: 30053671. DOI: 10.1016/j.ejca.2018. 05.017

24 Turner NC, Finn RS, Martin M, Im SA, DeMichele A, Ettl J, Dieras V, Moulder S, Lipatov O, Colleoni M, Cristofanilli M, Lu DR, Mori A, Giorgetti C, Iyer S, Bartlett $\mathrm{CH}$ and Gelmon KA: Clinical considerations of the role of palbociclib in the management of advanced breast cancer patients with and without visceral metastases. Ann Oncol 29(3): 669-680, 2018. PMID: 29342248. DOI: 10.1093/annonc/mdx797 
25 Verma S, Bartlett CH, Schnell P, DeMichele AM, Loi S, Ro J, Colleoni M, Iwata H, Harbeck N, Cristofanilli M, Zhang $\mathrm{K}$, Thiele A, Turner NC and Rugo HS: Palbociclib in combination with fulvestrant in women with hormone receptor-positive/her2-negative advanced metastatic breast cancer: Detailed safety analysis from a multicenter, randomized, placebo-controlled, phase iii study (paloma-3). Oncologist 21(10): 1165-1175, 2016. PMID: 27368881. DOI: 10.1634/theoncologist.2016-0097

26 Munsell MF, Sprague BL, Berry DA, Chisholm G and Trentham-Dietz A: Body mass index and breast cancer risk according to postmenopausal estrogen-progestin use and hormone receptor status. Epidemiol Rev 36: 114-136, 2014. PMID: 24375928. DOI: $10.1093 / \mathrm{epirev} / \mathrm{mxt} 010$

27 Gevorgyan A, Bregni G, Galli G, Ganzinelli M, Martinetti A, Lo Vullo S, Mariani L, Festinese F, Sottotetti E, de Braud F and Di Cosimo S: Body mass index and clinical benefit of fulvestrant in postmenopausal women with advanced breast cancer. Tumori 102(4): e11-14, 2016. PMID: 27174629. DOI: $10.5301 / \mathrm{tj} .5000515$
$28 \mathrm{Hu}$ W, Sung T, Jessen BA, Thibault S, Finkelstein MB, Khan NK and Sacaan AI: Mechanistic investigation of bone marrow suppression associated with palbociclib and its differentiation from cytotoxic chemotherapies. Clin Cancer Res 22(8): 20002008, 2016. DOI: 10.1158/1078-0432.CCR-15-1421

29 Myrand SP, Sekiguchi K, Man MZ, Lin X, Tzeng RY, Teng CH, Hee B, Garrett M, Kikkawa H, Lin CY, Eddy SM, Dostalik J, Mount J, Azuma J, Fujio Y, Jang IJ, Shin SG, Bleavins MR, Williams JA, Paulauskis JD and Wilner KD: Pharmacokinetics/ genotype associations for major cytochrome p450 enzymes in native and first- and third-generation japanese populations: Comparison with korean, chinese, and caucasian populations. Clin Pharmacol Ther 84(3): 347-361, 2008. PMID: 18231117. DOI: $10.1038 /$ sj.clpt.6100482
Received July 22, 2019

Revised August 11, 2019

Accepted August 21, 2019 